\title{
COUPLING OF THE FAST BOUNDARY-DOMAIN INTEGRAL METHOD WITH THE STOCHASTIC COLLOCATION METHOD FOR FLUID FLOW SIMULATIONS
}

\author{
JURE RAVNIK ${ }^{1}$, ANNA ŠUŠNJARA ${ }^{2}$, JAN TIBAUT ${ }^{1}$, DRAGAN POLJAK ${ }^{2} \&$ MARIO CVETKOVIČ $^{2}$ \\ ${ }^{1}$ Faculty of Mechanical Engineering, University of Maribor, Smetanova Ulica 17, SI-2000 Maribor, Slovenia \\ ${ }^{2}$ University of Split, Faculty of Electrical Engineering, Mechanical Engineering and Naval Architecture, Croatia
}

\begin{abstract}
In this paper we present the development of a Fast Boundary-Domain Integral Method for the solution of fluid flow and heat transfer problems. Adaptive cross approximation of integral matrices is performed on a hierarchically decomposed matrix structure. We couple the developed flow simulation algorithm with stochastic modelling of input parameters. In order to assess the influence of input parameters on the simulation results, we employ the stochastic collocation method as a wrapper around the deterministic code. We apply the developed numerical tool for the simulation of flow and heat transfer of nanofluids. Keywords: Boundary-Domain Integral Method, stochastic collocation method, sensitivity analysis, nanofluid, natural convection.
\end{abstract}

\section{INTRODUCTION}

The Boundary-Domain Integral Method [1] has been successfully used to solve several kinds of engineering problems. Its main drawback is the large computational resources required due to the appearance of fully populated matrices during the discretization procedure. In this paper we present the development of a Fast Boundary-Domain Integral Method [2], [3] for the solution of fluid fl ow an d he at tr ansfer pr oblems, wi th the aim to reduce computational requirements of the algorithm. The Navier-Stokes equations in velocity-vorticity formulation are considered. Diffusion and modified Helmholtz fundamental solutions are used to derive a Boundary-Domain Integral representation of the governing equations. We employ two discretization approaches. The vorticity transport equation is solved using a domain decomposition approach. This leads to a finite element type sparse structure of integral matrices and enables efficient use of computational resources. The kinematics equation, on the other hand, is solved on a single domain, which leads to full matrices of integrals. To mitigate the high computational resources required, we propose the use of a hierarchical decomposition of the domain, which leads to $\mathcal{H}$-matrix representation of integral matrices. Admissible matrix blocks are approximated using the adaptive cross approximation technique. Efficiency of the proposed acceleration strategy is discussed and an optimal compression ratio is found to exist. We define the optimal approximation ratio as the highest compression ratio, where the error introduced by the approximation is of the same order as other errors of the numerical scheme.

The reduced computational demands of the algorithm enables us to couple the developed flow simulation algorithm with stochastic modelling of input parameters. In order to assess the influence of input parameters on the simulation results, we employ the stochastic collocation method (SCM, [4], [5]) as a wrapper around the deterministic flow simulation code. In this way, we are able to propagate the uncertainty from input to output parameters. First, we identify the most important parameters using the "one-at-a-time" (OAT) principle and then, the full tensor SCM was used to assess the stochastic mean, variance and Sobol-like indices for sensitivity analysis [6]. 
Finally, we apply the developed numerical tool for the simulation of flow and heat transfer of nanofluids. A nanofluid is a term describing a dilute dispersion of particles in a fluid. The diameter of particles is in the order of ten nanometres. The particles are made of metal oxides, which enhances the thermal properties of the suspension. Natural convection in a cubical, differentially heated cavity is considered as a model problem for stochastic analysis.

\section{METHODS}

The velocity vorticity formulation of Navier-Stokes equations as a model for nanofluid flow and heat transfer was presented in Ravnik et al. [7]. In nondimensional setting, it reads:

$$
\begin{gathered}
\nabla^{2} \vec{v}+\vec{\nabla} \times \vec{\omega}=0, \\
\frac{\partial \vec{\omega}}{\partial t}+(\vec{v} \cdot \vec{\nabla}) \vec{\omega}=(\vec{\omega} \cdot \vec{\nabla}) \vec{v}+\operatorname{Pr} \frac{\mu_{n f}}{\mu_{f}} \frac{\rho_{f}}{\rho_{n f}} \nabla^{2} \vec{\omega}-\operatorname{Pr} R a \frac{\beta_{n f}}{\beta_{f}} \vec{\nabla} \times T \vec{g}, \\
\frac{\partial T}{\partial t}+(\vec{v} \cdot \vec{\nabla}) T=\frac{k_{n f}}{k_{f}} \frac{\left(\rho c_{p}\right)_{f}}{\left(\rho c_{p}\right)_{n f}} \nabla^{2} T,
\end{gathered}
$$

where $\operatorname{Pr}$ and $R a$ are the Prandtl and Rayleigh numbers respectively. The nanofluid is modelled using effective properties, such as: density $\rho_{n f}$, viscosity $\mu_{n f}$, heat capacitance $\left(c_{p}\right)_{n f}$, thermal expansion coefficient $\beta_{n f}$ and thermal conductivity $k_{n f}$. Models for these are listed in [8]-[9]. The solution of vorticity and energy transport eqns (2) and (3) used the sub-domain Boundary Domain Integral Methods, as proposed by Ravnik et al. [10].

For the solution of the kinematics eqn (1) for unknown boundary vorticity values, we use the following integral statement:

$$
c(\vec{\xi}) \vec{v}(\vec{\xi})+\int_{\Gamma} \vec{v} \vec{\nabla} u^{\star} \cdot \vec{n} d \Gamma=\int_{\Gamma} \vec{v} \times(\vec{n} \times \vec{\nabla}) u^{\star} d \Gamma+\int_{\Omega}\left(\vec{\omega} \times \vec{\nabla} u^{\star}\right) d \Omega,
$$

where $u^{\star}(\vec{r})=\frac{1}{4 \pi|\vec{r}-\vec{\xi}|}$ is the fundamental solution of the Laplace equation, $\Omega$ the domain, $\Gamma$ the boundary and $\vec{\xi}$ is the source point. Placing $\vec{\xi}$ into all boundary nodes and performing discretization leads to a full system linear equations. We approximate the matrices using the adaptive cross approximation (ACA) technique [11]. First, the matrices are subdivided using a recursive algorithm, which gives a hierarchical $\mathcal{H}$ matrix structure (Fig. 1) [12]. We defined an optimal compression ratio, where maximum compression is achieved at a negligible effect of simulation accuracy. Further details of the approach are given in [2], [3], [13].

In order to asses the influence of nanofluid parameters (density, viscosity, heat capacitance, nanofluid concentration and thermal conductivity) on the simulation results, we propose the use of a stochastic model, which consists of $d$ input parameters modelled as random variables $(\mathrm{RV})$, organised in input vector: $\mathbf{X}=\left[x_{1}, \ldots x_{d}\right]$. The SCM is based on the polynomial approximation of the considered output $Y$ in the $d$ dimensional stochastic space:

$$
\hat{Y}(\mathbf{X})=\sum_{k=1}^{N} L_{x}(\mathbf{X}) \cdot Y^{(k)},
$$

where $L_{k}(\mathbf{X})$ is Lagrange basis function and $Y^{(k)}$ is the output realization for the $k$ th input point. The interpolation points (SC points) in each dimension are calculated according to Gauss-Legendre quadrature rule. The interpolation in the multivariate dimension space is done by using the tensor product of basis functions. The stochastic mean and variance are calculated by following the formula from the statistics. 


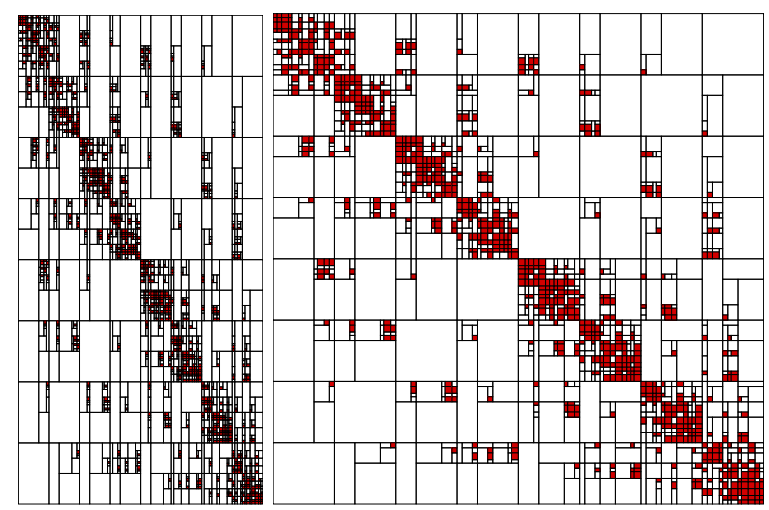

Figure 1: An example of domain-boundary (left) and boundary-boundary (right) $\mathcal{H}$ matrix structure. Red areas are inadmissible parts, where approximation is not performed, [2].

Sensitivity analysis based on variance decomposition in which the variance of a model is decomposed into terms depending on the input factors and their mutual interactions allows the computation of sensitivity indices of first and high order [5]. In practice, only first order indices are used:

$$
S_{i}=\frac{V_{X_{i}}\left[E_{X_{\sim i}}\left(Y \mid X_{i}\right)\right]}{V(Y)} .
$$

To capture the contribution of all terms which include the variable $X_{i}$, a total effect index is defined as follows:

$$
S_{T_{i}}=1-\frac{V_{X_{\sim i}}\left[E_{X_{i}}\left(Y \mid X_{\sim i}\right)\right]}{V(Y)} .
$$

A more simple way is a "one-at-a-time" (OAT) approach in which one input parameter is modelled as RV while the others are kept at nominal values. The impact of variables is determined by comparing their respective variances.

\section{RESULTS}

As a model problem we consider the development of 3D natural convection in a differentially heated cavity. Water with aluminium nanoparticles was the chosen nanofluid. We simulated the problem at different nanoparticle concentrations and temperatures differences for a steady state solution.

In the first step, 12 input parameters are modelled as random with uniform distribution set as $10 \%$ variation from nominal value: volume fraction, temperature difference, fluid viscosity, heat capacity, density, thermal conductivity, thermal expansion coefficient, characteristic dimension, particle heat capacity, density, thermal conductivity, thermal expansion coefficient. To reduce the dimensionality of a problem 12 univariate test cases (TC) were done following the logic of OAT principle. Stochastic simulations with 3 and 5 SC points in each dimension were run leading to total of 73 deterministic simulations. The convergence of the method was satisfactory. After comparing the variances from $12 \mathrm{TCs}$, five input variables are recognized as the important ones, while the others will be neglected in further computations. Those 5 input variables are: volume fraction, temperature difference, 


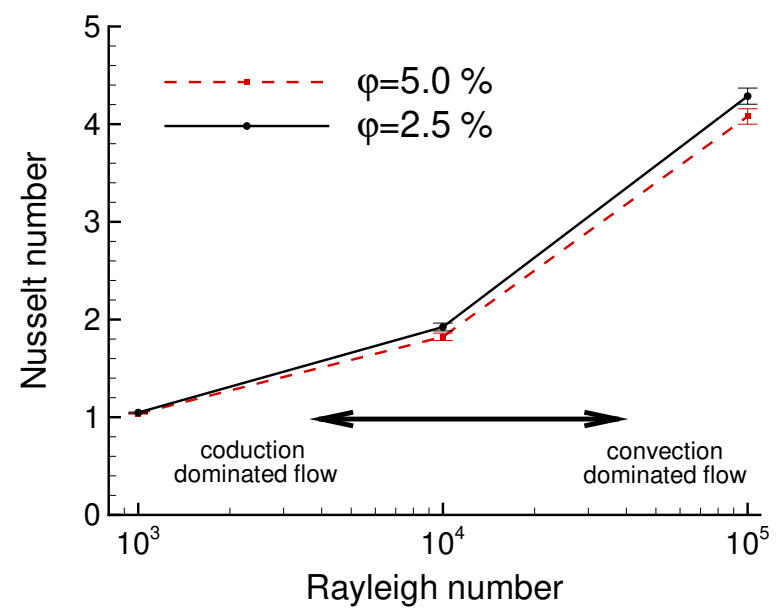

Figure 2: Heat flux variation due to uncertainty in input parameters. Results for two nanoparticle concentrations are shown.
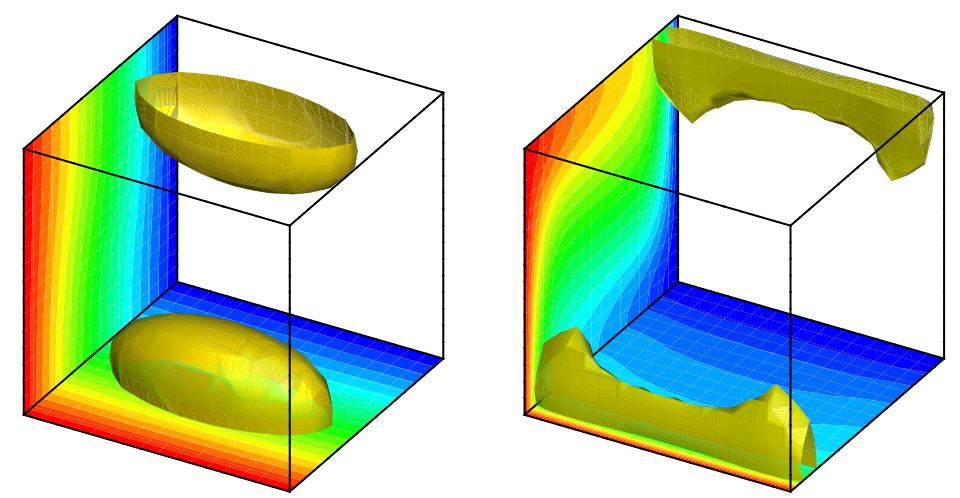

Figure 3: Isosurfaces indicating the volume where the greatest variation in temperature field occurs. Left panel: conduction dominated flow regime, $R a=10^{3}$, right panel: convection dominated flow regime, $R a=10^{5}$.

fluid viscosity, heat capacity and, thermal conductivity. In the second step a full tensor model with five random inputs and accuracy of 3SC was done.

In Fig. 2 we present the heat flux (expressed as Nusslet number) as a function of Rayleigh number. We observe that the uncertainty in input parameters is expressed more prominently in the convection dominated flow regime. Fig. 3 reveals areas in the flow domain where the greatest variation in the temperature field occurs. They are located at the top and bottom wall of the domain. In convection dominated flow regimes the areas of greatest variations are found along the edges of the domain.

\section{SUMMARY}

We presented an adapted cross approximation accelerated Boundary-Domain Integral Method for the simulation of nanofluids using the solution of the velocity-vorticity formulation 
of Navier-Stokes equations. The developed algorithm was coupled with a stochastic input parameter sensitivity analysis. We have successfully shown that acceleration of the boundary domain integral method enables performing larger simulation sets, which are needed to consider stochastic analyses.

\section{REFERENCES}

[1] Škerget, L., Hriberšek, M. \& Kuhn, G., Computational fluid dynamics by boundary domain integral method. International Journal for Numerical Methods in Engineering, 46(8), pp. 1291-1311, 1999.

[2] Ravnik, J. \& Tibuat, J., Fast boundary-domain integral method for unsteady convectiondiffusion equation with variable diffusivity using the modified Helmholtz fundamental solution. Numerical Algorithms, 2019. https://doi.org/10.1007/s11075-019-00664-3.

[3] Tibaut, J. \& Ravnik, J., Fast boundary-domain integral method for heat transfer simulations. Engineering Analysis with Boundary Elements, 99, pp. 222-232, 2019.

[4] Poljak, D., Šesnić, S., Cvetković, M., Šušnjara, A., Dodig, H., Lalléchère, S. \& El Khamlichi Drissi, K., Stochastic collocation applications in computational electromagnetics. Mathematical Problems in Engineering, 2018. https://doi.org/10.1155/2018/1917439.

[5] Saltelli, A., Annoni, P., Azzini, I., Campolongo, F., Ratto, M. \& Tarantola, S., Variance based sensitivity analysis of model output. Design and estimator for the total sensitivity index. Computer Physics Communications, 181(2), pp. 259-270, 2010.

[6] Ravnik, J., Susnjara, A., Tibaut, J., Poljak, D. \& Cvetkovic, M., Stochastic modelling of nanofluid heat transfer. 4th Workshop UNEMA, Split, Croatia, 2018.

[7] Ravnik, J., Skerget, L. \& Hribersek, M., Analysis of three-dimensional natural convection of nanofluids by BEM. Engineering Analysis with Boundary Elements, 34(12), pp. 1018-1030, 2010.

[8] Khanafer, K., Vafai, K. \& Lightstone, M., Buoyancy-driven heat transfer enhancement in a two-dimensional enclosure utilizing nanofluids. Int J Heat Mass Transfer, 46, pp. 3639-3653, 2003.

[9] Oztop, H.F. \& Abu-Nada, E., Natural convection of water-based nanofluids in an inclined enclosure with a heat source. Int J Heat Fluid Flow, 29, pp. 1326-1336, 2008.

[10] Ravnik, J., Škerget, L. \& Žunič, Z., Velocity-vorticity formulation for 3D natural convection in an inclined enclosure by BEM. International Journal of Heat and Mass Transfer, 51(17-18), pp. 4517-4527, 2008.

[11] Bebendorf, M., Approximation of boundary element matrices. Numerische Mathematik, 86(4), pp. 565-589, 2000.

[12] Hackbusch, W., A sparse matrix arithmetic based on H-matrices. Part I: Introduction to H-matrices. Computing, 108, pp. 89-108, 1999.

[13] Tibaut, J., Škerget, L. \& Ravnik, J., Acceleration of a BEM based solution of the velocity-vorticity formulation of the Navier-Stokes equations by the cross approximation method. Engineering Analysis with Boundary Elements, 82, pp. 17-26, 2017. 